\section{THE CROONIAN LECTURE}

\author{
PREVENTIVE INOCULATION. \\ Delivered before the Royal Society. \\ BY M. R OU X \\ ON BEHAIF OF
}

M. LOUIS PASTEUR. Membre de l'Institut.

Introduction.-Jennerian Vaccination.-The Discovery of the Artificial Attenuation of Tirus.-Pasteur's Researches on Ferments. - Researches on Chicken Choiera.-Attenuating Infuence of the Air.-Anthra.x Virus: its Attenuation or Intensification.-Preventive Inoculation for Rabies.-Statistics of Treatment at the Pasteur Institute-The Poisons Manufactured by Microbes.The Physiological Question.- The Action of Phagocytes.-The Search for Antidotes.

Gentlemen,- In the year 1881, M. Pasteur laid before the members of the International Congress assembled in London an account of recent researches carried on in his laboratory, on the subject of preventive inoculations for chicken cholera and splenic fever. Since that time nearly eight years have elapsed, and we may ask ourselves what has become of the work then begun : has it fulfilled its promise, and what pluce have the new principles which it involved taken in the science of to-day? It was on these questions that M. Pasteur intended to have spoken to you this afternoon, but the state of his health did not permit of his availing himself of the honour done him by the President and Council of the Royal Society in asking him to give the Croonian lecture this year. $\mathrm{He}$ therefore proposed that I should speak in his name, though personally I cannot hope to speak as he would have done of the preventive inoculations which he himself thought out and initiated. My sole title for addressing you is that of my being M. Pasteur's collaborator, having had, in fact, with Messrs. Chamberland and Thuillier, the honour of being associated with him from the commencement of his researches upon the prevention of contagious diseases, and, further, that I have been an eye-witness of everything which I shall lay before you.

Most infectious diseases never recur, and thus small-pox, measles, and typhoid fever rarely occur more than once in a lifetime. Further, a first attack of an infectious disease, even though a slight one, renders us safe from these diseases for a certain time; and it is this fact, coupled with the non-recurrence of infectious maladies, which has led to the discovery of preventive inoculation.

Instead of waiting till we are struck down unawares by a sudden attack of the malady, frequently during an epidemic of high fatality, and under conditions very unfavourable to our power of resisting it, we now seek to meet it at some favourable moment, and guarded by all those precautions which we know are capable of greatly diminishing the danger. In the place of natural, that is ordinary, infection, unforeseen and orer which we have no control, we have now substituted a mode of artificial infection, prepared in such a way as to ensure exemption with as little risk as possible.

It was against small-pox that, for the first time, preventive inoculations were had recourse to. An involuntary experiment, and one, unhappily, too often repeated, had shown that the liquid of the small-pox pustule is virulent, that is to say, that this smallpox lymph,introduced into the body through a wound in the skin, has the power of communicating the disease to a person who has not previously suffered from it. Inoculation with small-pox was, therefore, easy; all that was required for its production being a prick from a lancet charged with small-pox pus. It was, therefore, the custom to endeavour to find cases of mild small-pox, from the pustules of which a virus was taken supposed to be nonmalignant, but yet capable of subsequently rendering exempt against the virulent disease those who were inoculated by such a mild virus. It is well known to you how widely such inocula- tions spread, though far from being of an innocent character, for the inoculation which was supposed to give the disease in a mild form often produced it very severely, and 'sometimes even the inoculation was the cause of death.

How great, therefore, was the progress made by Jenner in replacing inoculation by vaccination, that is to say, in substituting for a severe illness one which is invariably insignificant, and yet is an efficient protection against infection by small-pox !

Although from the beginning of this century we have enjoyed the inestimable benefit of Jenner's vaccination, we still have not yet completely fathomed its meaning. What is the relation between vaccination and small-pox? Why does the vaccine disease of the horse and the cow, inoculated into man, render him exempt from small-pox? Is the virus of vaccine merely that of smallpox modified, or are vaccine and small-pox two different maladies?

It would have seemed that these questions were easy to resolve, as both small-pox and vaccine admit of experimentation upon them; yet, though always under discussion since Jenner's time, they yet remain without any definite solution having been arrived at. Jenner's great discovery, which seemed to open so wide and hopeful a horizon, has remained hitherto a solitary fact in medicine. Born of a happy observation, marvellously developed by a genius as patient as it was penetrating, it was at the time of its birth so far in advance of the medical science of the time that even now, after all the progress which has been made during the last seventy or eighty years, we can but suspect its real interpretation. Jenner thus demonstrated to us by cne remarkable example that it is possible to protect ourselves from a mortal malady by inoculation with a trivial one; but he gave us no general method leading to the prevention of other infectious diseases.

The discovery of the power of artificially attenuating-that is, weakening-a virus does, on the contrary, furnish us with a veritable method of protective inoculation, and it is one which has given us an uninterrupted series of good results, though this invention dates from but a very few years back, like all other recent progresses in our knowledge of virulent diseases, it found its origin in M. Pasteur's researches on ferments. In revealing to us the nature of ferments, he taught us that of the poison of infoctious diseases. Like the yeast of alcoholic and the yeast of lactic fermentation, viruses are living beings-microbes, as they are now called-and, just as the development of yeast in a sugary liquid produces alcoholic fermentation, so that of microbes in the tissues of the body produces the phenomena of infectious disease. The process which has enabled us to obtain the culture of microbic ferments in a state of purity is the same which has enabled us to obtain pure cultures of microbic virus outside of the body.

The indispensable condition of success in these cultivations is that of absolute purity - that is to say, the avoidance of the introduction of other foreign germs which everywhere surround us. For this purpose we have now arranged a definite technigue, strict but at the same time very simple. As the elements of the virus are living beings which can be kept in artificial cultivations, and as they are only distinguished from other lowly organised beings and plants by their property of invading the bodies of men and animals, the question naturally presents itself, Would it not be possible to modify them by cultivation in the same way that other plants are modified? Could they not, for example, be thus robbed of those qualities which make them formidable? To modify a virus by special modes of cultivations-such was the idea of M. Pasteur; a fruitful idea, from which have sprung those discoveries which I now propose to lay before you.

It was in studying a malady called "chicken cholera" that $\mathrm{N}$. Pasteur for the first time obtained by this means an attenuated virus. This disease is so fatal to fowls, pigeons, and birds in general that it has been given the name of cholera. It is caused by the development in the bodies of those attacked of a very small microbe, shaped like a small rod with rounded ends, and almost as wide as long. The photograph projected on the screen shows us the image of a drop of blood taken from a fowl which succumbed to the natural disease. You see between the globules of the blood the little rods which are the cause of the disease. It is, however, not the blood alone in which the microbe is found; all the tissues are invaded by it. The intestines contain a great quantity, so much so that the dejecta of the sick fowls are able to spread the malady, and it is in pecking upon the contaminated ground that healthy birds are infected.

If a minute drop of blood from a fowl which has just died 
of the malady be introduced under the skin of a healthy fowl, the animal inoculated soon falls ill, ceases to eat, its feathers are erected, its wings hang down, and it seems oppressed with unconquerable somnolence. It soon dies, sometimes in less than twelve hours. The blood of the bird which has thus died from experimental inoculation is found to be swarming with the microbe, exactly like that of the fowls which die after natural infection. It seems, then, that chicken cholera is a contagious disease capable of inoculation, and in which the virus is principally contained in the blood of the animals attacked by it. The culture of the microbe which is so easily accomplished in the blood of the animals can also be carried on artificially.

If with proper precautions we inoculate chicken broth slightly " alkalised," and perfectly limpid, with a drop of the blood, and if we then place the bottle in a stove at $35^{\circ} \mathrm{C}$, we shall find after some hours that the broth is turbid, and that this is due to the development of the little chicken-cholera microbe. Under the microscope we shall see that each smallest drop of this bouillon contains an innumerable swarm of motionless microbes, like those contained in the blood which served as "seed." An infinitesimally small quantity of this first culture placed in a new bottle will give a second culture, and by successive cultivations as many successive generations of our microbe as we wish for can be obtained. Each drop of these cultures, even up to the twentieth, would kill with all the signs of cholera, quite as surely as the first, any fowl which was inoculated by it. This experiment affords a decisive proof that the virus of the malady is without doubt the microbe found in our cultures, and as we now know how to prepare under absolutely certain conditions as large quantities of the virus as we wish for, we have all the means at hand for the study of this disease.

If we expose at a temperature of $33^{\circ} \mathrm{C}$ to the contact of the pure air which penetrates through the cotton wool stopper of the culture flask one of these cultivations which is so active that a drop of it would kill any fowl into whom it was inoculated, and if each week we extract a small quantity of the contents of the flask and try its virulence upon healthy fowls, we observe the following changes: During the first week of the experiment all the fowls inoculated die, but after a longer time a change sets in in the degree of virulence. Not all the fowls now die when a certain quantity of this longer preserved culture is injected under their skin. Some recover after having been very ill. As time passes the strength of the virus is still more diminished, and the number of fowls which recover increases. At last, in continuing the experiment, the moment comes, say, for example, after it has remained for two months in the incubator, when this virus, at first so deadly, not only will not kill a single fowl, but causes them apparently no inconvenience whatever; and yet the virus is not dead, for it still grows in fresh nutritive substance in which it may be sown. But in this new culture none of the former virulence returns. The daughter cultures have exactly the same effect upon the fowls as the mother culture had at the time when it furnished the fresh seed. 'The new property of the virus, therefore, that of harmlessness for those animals for which it was formerly so fatal, can be perpetuated for successive generations. By making such cultivations we shall obtain at their respective dates a whole series of kinds of virus of diminishing activity capable of giving to the animals either a fatal malady, a dangerous malady, a serious malady, or one wholly inoffensive.

To what cause is this gradual diminution of the malady due? To the continued action of the oxygen of the air. If, instead of making the cultivation in a bottle where the renewal of the air is possible, we were to make it in a closed tube only containing a small quantity of air, the microbe would soon consume all the oxygen, and would cease to grow, for oxygen is a necessity of its development. In a tube deprived of air it cannot grow; it remains alive in it for a very long time, as one can satisfy oneself by sowing in aërated bouillon the small culture formed in these sealed tubes. After being preserved for a year in this sealed tube it still gives cultures which are as active as a recent culture from the blood of a fowl just dead of chicken cholera.

We see, therefore, that the diminution in virulence which takes place in those cultures exposed to the air is dependent on that exposure, and not on the length of time the microbe is kept.

Gentlemen, what results were won, what new ideas gained by this single experiment on the culture of the microbe of chicken cholera? By it M. Pasteur showed us that the viruses of infectious diseases are no more the unchangeable entities they were before imagined to be. He taught us that, like all other living beings, the microbic virus is susceptible of modifications which heredity perpetuates, that it is above all the virulent character which becomes modified, and finally that this modification can be produced artificially and regulated according to the wishes of the experimenter. By this experiment M. l'asteur established the attenuating influence which the air possesses; at the same time he explained how it is that the activity of a virus, under natural conditions as seen in epidemics, is preserved or exhausted, and how the same malady may be sometimes malignant, sometimes light.

We have seen how fowls inoculated with attenuated cholera virus, suitably chosen, took the light form of the malady and soon recovered. If, now, you inoculate these recovered fowls with blood from a fowl dead of the virulent disease, and at the same time you inoculate a number of fresh fowls, you will find that all the fresh fowls will die, while those previously inoculated with attenuated virus will resist the disease. They will merely have a passing illness, which soon disappears. The inoculation with the attenuated virus has rendered them exempt from the fatal form of the disease; it has given them immunity; and if, in the same animals, you make successive inoculations of increasing intensity, you will make them refractory to cholera to such an extent that you may inoculate them with the most virulent blood, under conditions where they would be exposed to the most intense natural contagion, and they will not experience the least inconvenience from it, and it will be impossible to kill them by this illness, which was formerly so formidable.

The attenuated virus therefore discovered by M. Pasteur is quite as efficacious against chicken cholera as Jenner's vaccination against small-pox. But while we are ignorant of the relations between small-pox and vaccine, none of the relations between the vaccine virus of fowl cholera and the virulent virus are hidden from us.

This plan, moreover, is not only successful in the case of chicken cholera, but constitutes a definite method of attenuation of virus, the value of which has been proved by the production through its means of the vaccine of another malady more interesting than fowl cholera, as it is both a scourge for cattle and can also be transmitted to man. I mean splenic fever, anthrax, or charbon, for it is by the regulated action of hent and air upon the anthrax virus that the vaccine of this malady has been obtained; but in the case of anthrax a difficulty arose which was not present in that of chicken cholera.

The anthrax virus is found in the blood of animals which have just died of the disease. Cultivated in veal broth slightly alkaline it forms a culture resembling cotton down swimming in a clear liquid. This down is formed by long and interwoven filaments, as you see them in the photograph. In the interior of these filaments, after some hours, you see bright spots beginning to appear, the outline of which become more and more distinct. These brilliant spots are the germs or spores of the bacillus discovered by $M$. Koch ; these spores are the veritable seeds of the microbes, and as grains of corn, for example, offer more resistance to heat and dryness than does the growing corn, so the spores can bear without perishing a temperature of $90^{\circ} \mathrm{C}$., and the action of a number of agents which would kill the bacteride in its filament stage. The spore is thus the resistant condition of the microbe of anthrax, and wherever it finds conditions favourable to germinating, either in the body of an animal or in some artificial nutritive culture, it will give forth filaments, and these in their turn will produce new germs.

If we expose the anthrax bacillus to a temperature of $38^{\circ} \mathrm{C}$ and the contact of the air, as we did the microbe of fowl cholera, its virulence will not diminish. Even at the end of a long period it would still kill all the men and animals into whom it was inoculated. It consequently appears that the oxygen of the air is in this case unable to exert its attenuating influence, because the spores which are formed during the first hours of the culture are able to resist its action. In order, therefore, to repeat the conditions analogous to those which were successful in the chicken cholera, we must first prevent the bacillifrom producing spores. The way to do this is by cultivating anthrax not now at a temperature of $35^{\circ}$, but of $42^{\circ}$ to $43^{\circ} \mathrm{C}$. Under these conditions the bacilli develop, producing the filaments, but no spores. If we try every three days, for example, the intensity of the virulence of a culture thus made at a high temperature, and in which no spores are formed, by inoculating it into sheep and rabbits, we shall find that in the first days of the experiment all the inoculated animals succumb; then that the virus becomes less active, and the sheep 
resist, while the rabbits still die, though after illnesses more and more prolonged. After a still longer period the culture made at $42^{\circ}$ loses its danger for the rabbit, but is still fatal to guineapigs and to mice. Finally the moment arrives when it is quite harmless even to these little rodents, very sensitive to the anthrax virus as they are. We here see, as with fowl cholera, the virus passing through all the stages of decreasing virulence and finally becoming harmless. The bacilli, which at a high temperature give off no germs, yet form them rapidly if cultivated at $30^{\circ}$ or $35^{\circ} \mathrm{C}$., and the spores which are then produced preserve the same degree of virulence as the filaments from which they were derived. It is, therefore, only necessary to draw off each day a little of the culture from the bottle at $42^{\circ}$, and to place it in bouillon at $85^{\circ} \mathrm{C}$., in order to have a series of cultures of graduated virulence and furnished with spores capable of fixing each of these special degrees of intensity. The sheep and oxen which receive these attenuated bacterides exhibit a passing fever, but if, later on, you inoculate them with virulent virus, it has no effect upon them. One has, therefore, only to choose among the degrees in this scale of virulence that which will give to the animal one is desirous of protecting from anthrax an illness slight but sufficient to ensure the desired exemption. In practice the vaccinations of oxen and sheep are done twice. The virus of the first inoculation is very attenuated, and is intended to prepare the animal for the action of the second and more energetic inoculation, which is prepared twelve days after the first. The whole difficulty in anthrax inoculation consists in the choice of these two viruses, and in keeping the relation between their degrees of virulence invariable. Everyone still has in his remembrance the striking demonstration of the efficacy of these preventive inoculations given at Pouilly-le-Fort in 1881. Five-and-twenty sheep, chosen promiscuously from among fifty, were inoculated with attenuated virus of anthrax, and afterwards with the virulent virus at the same time that twenty-five otherfresh ones were inoculated as a control experiment. The twenty-five vaccinated sheep remained healthy; the twenty-five control sheep died of anthrax. Demonstrative as this experiment was, it did not obviate violent attacks, the first of which was against the principle of the method.

The possibility of obtaining attenuation of the anthrax virus under the conditions laid down by $\mathbf{M}$. Pasteur was denied. It is, however, unnecessary to dwell upon this point, as it is admitted even by those who contested it, for example, Koch and others; and the attenuation of the bacilli cultivated at $42^{\circ} \mathrm{C}$. has become a classical fact. The answer to lay criticism is to be found in these tables, which give the number of animals inoculated in France since 1881.

TABLE I.-Animals Vaccinated against Anthrax.

\begin{tabular}{|c|c|c|c|}
\hline Years. & Sheep. & Oxen. & Mortality among Sheep. \\
\hline $\begin{array}{l}1882 \\
1883 \\
1884 \\
1885 \\
1886 \\
1887 \\
1888\end{array}$ & $\begin{array}{l}243,199 \\
193,119 \\
231,693 \\
280,107 \\
202,064 \\
293,572 \\
269,599\end{array}$ & $\begin{array}{l}22,916 \\
20,501 \\
22,616 \\
21,073 \\
22,113 \\
42,538 \\
34,464\end{array}$ & $\begin{array}{l}1.08 \text { per cent. } \\
0.77 \quad ", \\
0.97 \quad ", \\
0.90 \quad ", \\
0.75 \quad "\end{array}$ \\
\hline
\end{tabular}

The mortality among sheep before the preventive vaccination for anthrax was 10 per cent.; since that period it has fallen to less than 1 per cent.

It shows how firmly established these inoculations are in agricultural practice, while the rise in their number is the most convincing proof of their efficacy. The farmer, of course, cares nothing for scientific discussions. For him, preventive vaccination is judged entirely from the standard of profit and loss; and in eight years he has had ample opportunity of coming to a definite conclusion on this point. The mortality among sheep in the anthrax districts has gone down from 10 per cent. to 1 per cent. Insurance companies insert a clause in their agreements making preventive inoculations of the insured cattle obligatory; and in Austria, Italy, and Spain the vaccinations are beginning to be widely adopted. What better arguments could be adduced to prove the great service which the discovery hus rendered to agriculture? But I do not desire to dwell on this point, and will proceed, having already discussed the attenuation of the virus, to consider the fact of its return once more to the virulent condition.

If in Nature we were to come across one of these attenuated bacteria which we have learnt to prepare, and which are so harmless as to be incapable even of killing a mouse, it would clearly be impossible for us to recognise in this inoffensive microscopical object a descendant of the terrible bacillus anthracis. To do so it would be necessary to have watched it in its stages of gradual attenuation. It is, however, possible (always supposing that the process of attenuation has not been carried ton far) to make the bacillus reascend the steps of virulence down which it has come, and so to render it once more virulent.

We have stated that it was not capable even of killing adult mice; but let us inoculate with it a very young mouse, only one day old. This young mouse will be much more sensitive to the action of the virus than the grown up one would be, and it will die in a few days. In developing itself in this young mouse, the bacillus will have recovered a portion of its old fitness for life. in living surroundings; and the blood of this first mouse introduced into that of one a little older will cause its death, and thus proceeding step by step from the youngest mouse to the oldest, it will gain power to kill first old mice, then guinea-pigs, rabbits, sheep, and, last of all, oxen, which are among herbivorous animals the most callous to the action of anthrax.

Thus we see that we can increase the virulence as easily as we can diminish it; and that it manifests itself by the increasing power of the microbes to germinate in the bodies of living animals, a power which can thus be either acquired or lost under respectively appropriate conditions. That this increase of virulence which we have thus excited is going on in Nature we can well imagine; and that a microbe, at first harmless for a particular kind of animal, should afterwards become dangerous to it. Given that some fortuitous circumstance should have introduced it into an animal with but feeble power of resistance, and it will grow there. This first cultivation will adapt it to parasitic life; it will pass out ready to develop in an organism in which it could not previously have gained a foothold, and after several such passages it will become really formidable.

There is no rashness in believing that by such means in the course of ages new forms of virulence have been evolved, and that these experiments on the variation of virulence throw a flood of light on that most obscure of questions, the origin of new virulent diseases. For these results also explain how one and the same kind of microbe can produce such diverse morbid effects: how, in its active or virulent state, it causes a general disease, rapidly ending in death, and in its passive or attenuated condition produces nothing more than a special local lesion.

Other viruses have also been attenuated by this method of the action of the air upon the cultures. At the Congress held in Geneva in 1882, M. Pasteur cited a whole series of fresh examples. I shall, however, only speak to you of the attenuated virus of a special form of swine fever, a disease much dreaded by farmers, and known in France by the name of "rouget," as the sick animals are distinguished before death by red patches on the skin.

The microbe of rouget is a bacillus which is found in the spleen and the lymphatic glands of the swine which die of the disease. In cultivation it produces no spores, and is therefore specially amenable to the influence of the air, and, in fact, if left long enough exposed to its action, its virulence is totally destroyed. But there is a further method of attenuation discovered by MM. Pasteur and Thuillier, and which is of great interest, as it shows the changes which a virus may undergo in its passage through animals of different kinds. Rouget injected into a rabbit kills it within a few days. A small quantity of the "pulp" from the spleen of this rabbit inoculated into a second one will cause death still more quickly, and the disease can thus be passed through a whole series of rabbits. But the extraordinary point (discovered by MM. Pasteur and Thuillier) in these inoculations is that, as the strength of the virus increases for the rabbit it is diminished for the pig; so much so that, after a sufficient number of passages of the virus have been made through the rabbit it has become a vaccine for swine, able to confer upon them exemption from the fatal form of malady.

With this example before us, the question arose, What would happen with certain human diseases if we made them also pase through a great number of different kinds of animals? Is not this very process taking place in Nature, and does it not give fresh support to the idea that the vaccine of small-pox becomes modified by its passage through the horse and the cow?

1 This form is known in Ireland as "red soldier" and is not the same disease as the pneumono-enteritis called "swine fever" in this country.-Tr. 
After all this work upon the prevention of fowl cholera, anthrax, and swine fever had been accomplished, M. Pasteur devoted himself to the study of rabies. When, in 1880 , the study of this disease was begun in M. Pasteur's laboratory, the following facts concerning it were alone known: that it was an infectious disease; that the virus was contained in the saliva of rabid animals, and that it was transmitted through their bites. We knew also that the period of its incubation varied from some days to some months, and here our definite knowledge of its pathology ended. Many experiments, however, had been made on the subject, but two causes had rendered them especially difficult to carry out and their success uncertain.

Inoculation of the saliva of a rabid into a healthy animal does not always produce rabies, but often has no effect whatever. Among animals susceptible to the malady, some only become rabid after such a lapse of time that the prolonged waiting, combined with the uncertainty of the results, puts the patience of the experimenter to a most severe test. The saliva of a rabid animal affords an untrustworthy virus, because it contains a quantity of different microbes, which, when introduced under the skin contemporaneously with the virus of rabies, may prevent the development of this latter, and set up inflammatory processes originated by their growth. The first thing to do, therefore, was to find some source of the virus of rabies which should be uncontaminated by other microbes. All the symptoms of rabies arise from the disturbance of the nervous system; hence the idea that in that system the rabic virus was specially to be found presented itself to the mind. The previous attempts made, however, to show that the nervous system of a rabid dog was virulent were unsuccessful, because the manipulations to which the nervous matter was exposed in order to inoculate it introduced into it those other microbes which it was essential to exclude.

By inoculating with absolute purity from the spinal cord the brain or the nerves of an animal which had died of rabies, $M$. Pasteur demonstrated that the true seat of the virus was to be found in the nervous system. A portion of the nerve centres of a rabid dog injected subcutaneously into a healthy one will produce rabies more surely than the most active saliva. This demonstration enabled us to take a decided step forward in its study. The rabic virus being contained in the nervous centres, and the symptoms depending entirely upon that system, the idea naturally followed that the disease only shows itself when the nervous centres are attacked by the virus. Further, that the incubation period is governed by the time taken by the virus to travel from the point inoculated up to the cerebro-spinal axis and for its development therein. If, therefore, the virus be inserted directly into the nervous system, where it has to develop, the incubation ought to be shortened and the disease follow with certainty, because the virus can no longer be destroyed or diverted from its course during its long journey.

Gentlemen, experiment has fully confirmed this theory, as may be seen from the record of the first dog inoculated by trephining, in which the incubation period was reduced to fourteen days. In fact, any dog inoculated under the dura mater with a little of the spinal cord or brain of a rabid dog takes rabies with absolute certainty and within a period rarely extending beyond eighteen days. Thus we are delivered from the uncertainties belonging to subcutaneous inoculations and from the weariness of a long incubation period. After this experiment the study of the disease went rapidly forward; it was proved that the virus existed in the nerves and that by that route it travels from the original wound to the brain and spinal cord, and also that it can in some cases be conveyed by the circulatory system. It will be seen, therefore, that the manifestations of rabies may be varied, as the manifestations of the disease in the beginning will depend upon the particular region first encountered by the virus, and finally why it is that there are forms of the disease which until now were unrecognised, and which differ from the classic type. The operation of trephining is in itself harmless when performed with proper antiseptic precautions. It is as successful with the rabbit as with the dog. If inoculation by trephining is performed upon a series of rabbits from the spinal cord or medulla oblongata of an animal which has just died, and this process is continued with successive animals, we find that the duration of the incubation period, which was at first from fourteen to eighteen days, gradually diminishes. It becomes shorter and shorter as the number of passages of the virus increases, until, at the end of a hundred of the successive inoculations, it has gone down first to seven days, and finally to six. At that point it remains stationary, the rabic virus seeming then to have attained its maximum virulence for that animal (the rabbit), and the virus is said to be "fixed." It' is from this "fixed" virus that M. Pasteur obtains his vaccine for rabies by a process similar in several details to that already used for attenuation in chicken cholera, swine fever, and anthrax.

If in a bottle with an upper and lower tubulure containing below fragments of caustic potash and closed by a piece of cotton-wool there is placed a spinal cord of rabbit which has served in a passage series of inoculations, this cord, which contains a quantity of " fixed" virus, gradually dries preserved from dust and exposed to the contact of the air at a temperature of $25^{\circ} \mathrm{C}$, care being taken to keep it at this point. If each day we take a small quantity of this cord and inoculate on the surface of the brain of a rabbit, we shall perceive that as the cord becomes dry in the sterilised warm air so it loses its virulence. At the end of five days' drying it will only be capable of killing some few of the rabbits who have received it. At the end of fourteen days we find it absolutely innocuous after having passed down the scale of gradually diminishing activity during the preceding days.

Having now obtained our material containing the attenuated virus, if we each day inject subcutaneously into a dog a portion of the attenuated cord, crushed in water, taking care to begin the injection with the harmless fourteen days' cord, to go on on the second day with the thirteen days cord, then the third day with the twelve days' cord, and so on till we reach the cord at zero, or, in other words, spinal cord unattenuated, spinal cord which is deadly, this dog thus inoculated will not die, nay more, we may try him with the most active rabic virus inoculated into the brain and he will remain perfectly well, though we know that otherwise intracranial inoculation produces rabies without fail. There is, therefore, positive proof that the injections of the dried cord have produced exemption from the disease. The experiment may be repeated as often as you please, but the results remain the same. Dogs which have subcutaneously received the series of cords commencing from the fourteenth day cannot take the disease either from the bites of mad dogs or in any other way. The exempt condition has been obtained in one fortnight.

Usually rabies remains latent in a dog which has been bitten for a period generally exceeding one month. It might therefore be possible to profit by this long incubation period to give before its manifestation exemption from the malady. To elucidate this point, out of a number of dogs bitten by rabid dogs, or inoculated under the skin with rabic virus, some were preserved for control experiments, while the others were subjected to the preventive inoculation of the dried cord in augmenting degrees of virulence. Of these latter not one took rabies, while of the former a great number died with the characteristic symptoms of the malady. It was proved possible to prevent hydrophobia even after the bite.

Notwithstanding, however, the favourable results obtained upon animals, the application of the same method to man was undoubtedly a bold step. By what urgent solicitations and "medical advice" M. Pasteur agreed to take it is a matter of history. In July, 1885 , the boy Meister, terribly bitten by a rabid dog, was the first to undergo the anti-hydrophobic inoculation. It is a noteworthy date, and marks an epoch, not only in the history of the laboratory of $M$. Pasteur, but in that of Science herself. I shall not dwell upon that which is well known; how, after this first successful attempt, persons bitten came to the laboratory from all parts, nor how, since then, each month about 150 persons come to obtain the antirabic inoculations. Some among you have been present at these inoculations, and have seen how the emulsions of dried-that is, attenuated-cord are prepared to avoid the introduction of any alien germs. The injections are made in the side on the right and left alternately, and are repeated during fifteen days. For ordinary bites the inoculations start with the fourteen days' dried cord, and end with that of three days. For more dangerous wounds, those of the head and face, the number of inoculations is greater, and we proceed to the more recent cords more quickly, as it was soon discovered that these last-mentioned kinds of wounds required a more active treatment.

Since the commencement of the inoculations until now (March 21st, 1889) 6,870 persons have been treated in the Paris institute alone; and among them many were very severely wounded. The proof of the rabidity of the animal which gave the bite has been furnished either by experiment or by veterinary examination in 80 per cent. of the cases. The mortality among the persons bitten by indubitably rabid dogs and thus treated is about 1 per cent., a 
very low rate if compared with that of 15 per cent. which usually follows the bites of mad dogs. It is scarcely credible that this extremely small number of failures should have given rise to such violent attacks against this practice; the most contradictory accusations have been brought against it. It was declared to be inefficacious, that its good results were obtained by the treatment having been applied almost entirely to persons bitten by healthy dogs, that the statistics showed that as many persons had died in France of rabies since the introduction of M. Pasteur's system as before it. This last assertion was made by badly-informed persons, who took for complete statistics documents allowed to be insufficient even by the very individuals who published them. The proof of the efficacy of the system is to be found by examination of those cases where the rabidity of the attacking animal was incontestably, because experimentally, proved; and above all, from such of these cases as were bitten on the head and face. In this latter class alone, it is well known that the ordinary mortality is 80 per cent., whereas among those treated at the Pasteur Institute the mortality does not reach 4 per cent.

Other adversaries asserted that the treatment was dangerous, and increased the probability of death, and thus found themselves confronted with the singular paradox of a dangerous treatment enormously diminishing the death-rate of the disease, and with their previous assertion that the inoculations produced no result whatever. These last opponents were furnished in support of their contentions with the violence of their assertions alone, as they had made no experiments whatever.

Others followed, however, who contended that they had proved by experiment that the foundations themselves of the system were unsound, and that the antirabic inoculations could not give immunity to dogs. The fate of these pretended experiments is well known to you. They were shown, on unquestionable authoritythat of the English Commission charged with the inquiry into the inoculations - to be inexact. You know, gentlemen, of whom this Commission was composed, ${ }^{2}$ and in order to answer once for all the whole series of attacks, it is only necessary to recall their judgment as embodied in their report-namely, that $M$. Pasteur had discovered a method of prevention against rabies comparable to that of Jenner against vaccination. The absolute nullification of fatal results among persons bitten by mad animals and treated I do not, however, believe to be possible.

Nearly all the individuals treated who have succumbed to the disease developed it during the fortnight following the commencement of the inoculation, owing to the fact that in their case the virus passed probably by the blood-stream to thenervous centres immediately after the bite. Indeed, experience shows that the disease may break out from the first (?) to the eighteenth day after inoculation, and it also demonstrates that in cases of submeningeal inoculation it is extremely difficult to outstrip the disease, because the inoculation period is so short that attenuated cord inoculated subcutaneously and far from the central nervous system has no time to do its work. In such cases, therefore, of extremely short incubation period, it is possible that the treatment may fail. Fortunately, they are rare, even where the bites have been received in the face. As for the few cases which succumb even where the treatment has been completed and has apparently had sufficient time to produce its effects, it is extremely difficult to assign the true cause of such failures, but they may perhaps depend upon the special liability of the individuals in question.

The most remarkable point, however, in the whole discovery of this preventive inoculation against rabies is that it has been caried out, the virus itself being still unknown. Not only do we not know how to cultivate it outside the body, but, in allowing it to be really a microbe, we can but do so by analogy, for as yet no one has been able to isolate it. Notwithstanding this, however, it is daily being attenuated and made to pass through the various stages of virulence. Unable to cultivate the organism artificially in flasks and tubes, M. Pasteur has been obliged to do so in the rabbit, and so easily and with such perfect regularity are these cultivations in the living animal performed that they are ready each day for use in the inoculations at a specified time and in the condition of genuinely pure cultivations. There is no stronger example of the power of the experimental method applied to medical matters than this one of the prevention of a malady the absolute virus of which is still obscure.

Certain microbes-that, for example, of anthrax-grow with

2 The members of the Commission were Sir James Paget, Sir Joseph Lister. Drs. Brunton, Fleming, Quain, and Burdon Sanderson, Sir Henry Roscoe, and Mr. Horsley (Secretary). such tremendous rapidity in the bodies of animals, that at the time of their death their blood contains more parasitic elements than blood-corpuscles. The bacilli form occasionally capillary obstructions, and so act mechanically; but like all living cells, they have their vital products, and with such an enormous number of them it is easy to imagine that these very largely modify the nature of their surroundings. The bacillus of anthrax, which is specially greedy of oxygen, draws it from the blood-corpuscles, and produces asphyxia of the tissues; but the greatest source of danger from microbes is to be found in poisonous products which they manufacture. A striking proof of this fact is given us by the diphtheritic bacillus, which, notwithstanding that it grows not in the interior of the tissues, but on the surface of the mucous membrane-outside, as it might be called, the body-yet causes death, and sometimes with a frightful rapidity. In this case there is no invasion of the body nor struggle between the cells, but simply poisoning by the products of a very active parasite growing on the border of the false membrane. It is difficult to find in the body of an animal which has just died of an infectious malady these poisonous products of which we have been speaking, as the complex surrounding of tissues is unsuitable for such researches, and the poisons being only present in very small quantities, the animal during life partly eliminated them from its system; it is therefore in the cultivations in flasks and tubes that we must endeavour to find these products of the pathngenic activity of the microbes.

The first experiments made on this subject were originated by M. Pasteur. In order to find out what the action of the products of the cholera microbe really was upon fowls, M. Pasteur injected into them a large quantity of a culture which had been absolutely purified from the microbes by filtering it through porcelain. The fowl into which this liquid, absolutely free from living virus, was injected, became sleepy, its wings hung down, its feathers stood up, and in fact for several hours it displayed all the symptoms of the disease, after which it recovered. We thus see that the chemical products obtained during cultivation of the microbe are able of themselves to cause the symptoms of the disease, and it is therefore very probable that they are really manufactured by the microbe in the body itself of a fowl attacked by cholerd. It has since been shown that many of the pathogenic microbes manufacture these poisonous products, and the microbes of typhoid fever, Asiatic cholera, blue pus, acute experimental septicæmia, and of diphtheria, all belong to this class. The cultivations of the bacillus of diphtheria in particular become, after the lapse of a certain time, so charged with the poisonous substance that an infinitesimally small dose of it causes death to the animals, with all the characteristic signs observable after inoculation with the microbe itself, no one sign being wanting to complete the resemblance, even down to the gradual encroachment of paralysis where the dose has not been sufficiently strong to ensure a speedy death. In infectious maladies, therefore, the cause of death is poisoning, and the microbe is not merely the means of spreading infection, but is also the make $r$ of the poison.

By introducing little by little into the bodies of animals these chemical substances produced by pathogenic microbes, such, for instance, as that of acute septicæmia, in such a manner as to avoid causing speedy poisoning, but so as to gradually accustom the animal to its presence, it becomes refractory not only to toxic doses, which would have originally caused death, but also even to the microbe itself; and the immunity which hitherto we could only give by the introduction of a living virus into the body we can now effect by the introduction of a chemical substance into the tissues, and these vaccine substances are exactly those which we have observed in infectious diseases as being the cause of death. In large quantities they kill, in small they confer immunity. These experiments on vaccination by means of soluble substances without microbes have been successful in various maladies, and we may be allowed to hope that their field of utility will become much wider.

I would here recall to you the works of Salmon, on Cholera; of Toussaint, Chauveau, Wooldridge, Chamberland, and myself, on Anthrax; of Charrin, on the Pyocyanic Bacillus Disease; Chamberland and myself, on Acute Septicæmia ; Beemer, Brieger, Chantemesse and Vidal, on Typhoid Fever; and of myself on Symptomatic Anthrax, which have established the principle of vaccination by chemical substances; so that we now see it may be possible to protect ourselves from one malady by means of another, for which purpose it is only necessary that the microbes of the two maladies should manufacture similar chemical substances. The question 
now arises, Has the animal, the recipient of a sufficient dose of these products, become refractory in consequence of their being present in the tissues, and thus preventing the growth of the microbe? Upon this point, while we do know that in cultivation the growth of certain microbes is arrested by the accumulatio 1 of the products which they form there, we must carefully avoid forming a definite opinion as to what happens in the living body upon the basis of phenomena which have been observed to take place in culture tubes. For example, if we take a little blood from a sheep which has been rendered refractory to anthrax, and place in it anthrax bacilli, they will grow there rapidly and abundantly, thus showing that there is in the blood of this protected animal no substance capable of destroying the life of the bacteria. This experiment is, of course, an extremely crude one, since from a chemiaal point of view there is an enormous difference between blood while still retained within the living vessels, and the same blood drawn from the body and placed in a culture flask. If any positive result were obtained, it could only be from an absolutely enormous chemical change in the composition of the tissues.

A more delicate experiment may be performed by injecting into the anterior chamber of the eye of a protected sheep a few virulent bacilli, which, while growing well in the aqueous humour, will confine themselves to that special spot. It is, therefore, evident that in this aqueous humour, notwithstanding its being a part of the animal, and consequently, of necessity, participating in its chemical modification, there is no substance present capable of resisting the local development and vital activity of the anthrax bacillus.

Besides the chemical, we have also the physiological, question to deal with, as may be seen from the following experiment. If the virus of " quarter evil," that is, charbon symptomatique, be injected into the thigh of a rabbit, an animal which is by nature refractory to the disease, no characteristic tumour will develop, and its immunity appears to be absolute. But if now any lesion be first produced in the tissues, either by a blow or by the injection of some caustic substance, and the inoculation be made at that point, then an anthrax tumour will soon appear, and the rabbit, though habitually exempt from the disease, may chance in this manner ultimately to succumb to it, the reason being, of course, that the injured tissues have formed an inert soil for the microbe to start its growth without hindrance. The immunity, therefore, of a rabbit from "quarter evil" does not arise from its possessing in its body any simple chemical substance inimical to the cultivation of the virus, as we see that it is only by artificially causing necrosis of the tissues that the virus is able to gain a footing in it.

What then, does happen on the injection of active virus into an animal which is rofractory? What becomes of the microbes? M. Metschnikoff has taught us that they are soon destroyed, and that the foremost agents of the destruction are the white corpuscles or phagocytes, which swallow up the microbss and digest them, while, on the other hand, the white corpuscles in the bodies of nonrefractory animals do not swallow the microbes, or that, if they attempt to do so, the latter develop notwithstanding.

Any satisfactory explanation of the problem of immunity should comprise all these facts, and should be based upon the relative importance of the action of the chemical products and the resistance of the tissues. I believe that the best explanation, in fact, consists in considering immunity as the result of the habituation of the cells to the poisons secreted by the microbes.

When the virus begins to develop in the body of an animal subject to the malady it forms its poisons, and when the white corpuscles begin to struggle against them their activity is arr :sted by this toxic production. The microbe continues to develop and the malady progresses. But in the case of an animal rendered refractory by previous injection of the soluble substances or by inoculation of attenuated virus, the corpuscles have already got accustomed to the microbic poison, and since the small doses given at the beginning of the cultivation of the virus do not impede their activity, they commence the struggle and devour the parasites. But if, as in the experiment with "quarter evil," some cause should privent this corpuscular intervention, the cultivation of the microb ; would succeed, and in this local centre there would soon be a sufficient quantity of poison to render the corpuscles powerless, notwithstanding that these have been previously accustomed to it, or possess natural powers 0 ? resistance against it.

There is no means of habituating the system to large doses. It is therefore in the time immediately following inoculation that the decisive battle is waged. Hence the great importance of clearly understanding the importance of the condition of the seat of inoculation, and of the quantity of virulent matter introduced. When once we are thoroughly acquainted with these poisonous substances formed by pathogenic microbes, we shall perhaps be in a position to find antidotes for them capable of paralysing their action even within the tissues themselves; but in offering this suggestion I perceive that I have deserted the domain of fact for that of bypothesis, and that it is therefore time I drew my remarks to a close.

It seems to me that the foregoing view of this wide question reconciles the various works upon it, which have been multiplied in the last few years, and though it is possible that it may be modified by time, one thing will remain unchanged, and that is the gratitude of all to him who by his studies of attenuated viruses and preventive vaccinations has enabled us to deal saccessfully with that problem of immunity which has hitherto remained impenetrable.

\section{THE CROONIAN LECTURES}

ON THE RELATIONSHIP BETWEEN

\section{CHEMICAL STRUCTURE AND}

\section{PHYSIOLOGICAL ACTION.}

Delivered before the Royal College of Physicians of London.

Br T. LAUDER BRUNTON, M.D., D.Sc.EDIN., LL.D.ABrer., F.R.S.

Fellow of the Royal College of Physicians; Assistant-Physician and Lecturer on Materia Medica at St. Bartholomew's Hospital.

INTRODUCTION.

Mr. President and Grntlemen,-Allow me before commencing these lectures to return yon my most sincere thanks for the honour you have done me in asking me to deliver them, for the stimulus you have given me to collate the facts relating to a subject of the greatest practical importance, and for the substantial aid which you have afforded me in doing it. The motto of this College "Art is long and Time is fleeting," as it has been translated by Longfellow, is one which, while it tends to repress too sanguine expectations of individual success in furthering medicine, is yet a strong incentive to individual effort, and its teaching has been well put by the same poet in the words:

\section{Let us then be up and doing With a heart for any fate \\ Still achieving, still pursuing, \\ Learn to lapour and to wait.}

The period of waiting on the advance of medicine has indeed been a long one, and in the Goulstonian Lectures which I had the honour to give in this place twelve years ago, I.tried to show to some extent why the progress of medicine has been so slow.

The Causes of Retardation.-Its advance has been mainly hindered, as I believe, because men have been guided in their practice more by their own fancies than by objective facts. I expressed a hope that the advance of medicine would become more and more rapid as the knowledge gained by close study of objective phenomena became more and more the basis of our practice. I also ventured to echo the prediction made by Dr. B. W. Richardson in 1868,1 that, ere long, we should be able to manufacture in our laboratories drugs which would produce in the animal body any effect that we might desire, without our having to obtain them, as one might say hap-hazard, from various plants, or other sources. AdVANCEMENT OF MEDICINE AND SURGRRY.

Ancesthetics.-Already we owe a great deal to chemistry for the potent medicines which it has prepared and supplied to us. Probably the two most striking advances which have been made in the healing art during the present century are the introduction of anæsthetics and of antiseptics. Anæsthetics have not only relieved the pains of labour and lessened the agony which occurs. in various diseases coming within the province of the physician, but they have taken away the terror formerly associated with surgical operations, and have allowed the surgeon, in place of try-

\footnotetext{
1 British Association Reports, 1868, p. 186.
} 